\title{
Avifauna en dos complejos de páramo de Antioquia, Colombia
}

\section{Avifauna of two paramo complexes in Antioquia, Colombia}

\author{
Sergio Chaparro-Herrera, Andrea Lopera-Salazar, Ana M. Gutiérrez-Zuluaga, Jefry Betancur, \\ Dariel Martínez Alvarado, Héctor Fabio Rivera Gutiérrez y Juan L. Parra
}

\section{Resumen}

Hicimos caracterizaciones rápidas de avifauna en dos complejos de páramo (Frontino-Urrao y Sonsón) en el departamento de Antioquia, Colombia, en el límite superior de bosques nublados, zonas de transición y páramos. Registramos 197 especies de aves (40 familias), de las cuales 7 presentan alguna categoría de amenaza, 1 está casi amenazada, 5 son endémicas, 15 casi endémicas, 4 son migratorias boreales y 1 es migratoria austral. Señalamos 12 especies de importancia, ya sea por su grado de amenaza nacional o por presentar ampliación en su rango de distribución geográfica o altitudinal.

Palabras clave. Aves. Cordillera Central. Cordillera Occidental. Ecosistemas de alta montaña. Frontino-Urrao. Sonsón.

\begin{abstract}
We characterized bird communities in two paramo complexes (Frontino-Urrao and Sonsón) in the department of Antioquia, Colombia, including the transition zones and the upper boundaries of cloud forests. We recorded 197 bird species ( 40 families), of which 7 presented some threat category, 1 is almost threatened, 5 are endemic, 15 near endemic, 4 boreal migratory species and 1 austral migratory species. We point out 12 species that are relevant, either due to their degree of national threat or because they represent geographic or altitudinal range extensions.
\end{abstract}

Keywords. Birds. Central Andes Mountain Range. Frontino-Urrao. High mountain ecosystems. Sonsón. Western Andes Mountain Range. 


\section{Introducción}

Colombia cuenta con el $50 \%$ de los páramos andinos, que ocupan un área aproximada de $29,000 \mathrm{~km}^{2}$, correspondientes al $2.5 \%$ del área continental del país (Sarmiento et al., 2013). Se reconocen 36 complejos de páramo, distribuidos a lo largo de las tres cordilleras y en la Sierra Nevada de Santa Marta (Sarmiento et al., 2013; Marín \& Parra, 2015). Colombia es, además, el sexto país con la mayor extensión de bosque nublado, con 15,228 $\mathrm{km}^{2}$ distribuidos en las tres cordilleras $\mathrm{y}$ en formaciones montañosas aisladas como las serranías de San Lucas, Baudó, La Macarena y la Sierra Nevada de Santa Marta (Mulligan \& Burke, 2005; Morales \& Armenteras, 2013). Estos ecosistemas ofrecen importantes servicios, como la regulación del agua y la retención de carbono, además de ser importantes refugios de biodiversidad (Morales et al., 2007; Sarmiento et al., 2013; Cabrera \& Ramírez, 2014; Marín \& Parra, 2015). Pese a su importancia, las zonas de alta montaña en general en el país se encuentran en un alto riesgo, debido principalmente al cambio en el uso de la tierra (por ejemplo, desarrollo de prácticas como la minería, la agricultura, la ganadería y el crecimiento poblacional), y al cambio climático (Rivera \& Rodríguez, 2011; Cabrera \& Ramírez, 2014; Marín \& Parra, 2015).

Caracterizamos la avifauna en dos complejos de páramo: Frontino-Urrao, al norte de la cordillera Occidental en el departamento de Antioquia, y Sonsón, en la cordillera Central en los departamentos de Antioquia y Caldas. Ambos complejos hasta la fecha han sido poco estudiados a nivel ornitológico, contando únicamente con datos de exploraciones realizadas en el páramo del Sol en 2004 (Flórez et al., 2004; Krabbe et al., 2005). Por lo tanto, se requiere evaluar el estado de las poblaciones de aves en estas localidades, para reconocer la importancia de estos ecosistemas en la conservación de la avifauna colombiana.

\section{Materiales y métodos}

Área de estudio. El complejo de páramo Frontino-Urrao está localizado al norte de la cordillera Occidental en los departamentos de Chocó (municipio del Carmen de Atrato) y Antioquia (municipios de Caicedo, Salgar y Urrao), entre los 3400 y 3930 m s. n. m.; comprende dos conglomerados, con una extensión de 13,921 hectáreas (Morales et al., 2007). De su extensión, 13,697 hectáreas corresponden a bosques, vegetación arbustiva y herbácea (Sarmiento $e t$ al., 2013). Dentro de este complejo es posible encontrar dos biomas generales: orobiomas del zonobioma húmedo tropical, que incluyen el orobioma andino de la cordillera Occidental y el orobioma de páramo cordillera Occidental, y un gran bioma de ecosistemas transformados que incluye el bioma de piso bioclimático páramo (Morales et al., 2007). En este complejo se seleccionaron para muestreo cinco localidades (Tabla 1, Figuras 1 y 3 ).

El complejo de páramo de Sonsón está localizado en la cordillera Central, entre los departamentos de Antioquia y Caldas, y tiene una extensión de 8707 hectáreas, de las cuales 7903 hectáreas corresponden a boques, vegetación arbustiva y herbácea, entre otros tipos de vegetación (Sarmiento et al., 2013). Dentro de este complejo es posible encontrar dos biomas: orobiomas del zonobioma húmedo tropical, que incluyen el orobioma andino y altoandino de la cordillera Central y orobioma de páramo de la cordillera Central, y un gran bioma de ecosistemas transformados, que incluye el bioma piso bioclimático páramo (Morales et al., 2007). Dentro de este complejo se seleccionaron para los muestreos cinco localidades (Tabla 1, Figuras 2 y 3 ). 
Tabla 1. Ubicación y mes de visita de cada localidad muestreada dentro del complejo de páramos Frontino-Urrao y Sonsón, Colombia.

\begin{tabular}{|c|c|c|c|c|c|c|}
\hline $\begin{array}{c}\text { Complejo } \\
\text { (sigla) }\end{array}$ & $\begin{array}{l}\text { Localidad } \\
\text { (sigla)/mes }\end{array}$ & Municipio & Departamento & Latitud & Longitud & $\begin{array}{c}\text { Rango de } \\
\text { elevación } \\
\text { (m) }\end{array}$ \\
\hline \multirow{5}{*}{$\begin{array}{l}\text { Frontino-Urrao } \\
\qquad(\mathrm{F}-\mathrm{U})\end{array}$} & Pená (P)/marzo & \multirow{2}{*}{ Abriaquí } & \multirow{9}{*}{ Antioquia } & -76.0358 & 6.509 & $3358-3424$ \\
\hline & La Horqueta $(\mathrm{H}) /$ julio & & & -76.1326 & 6.5689 & $2200-3600$ \\
\hline & El Junco (J)/marzo & Caicedo & & -76.0601 & 6.5023 & $2700-3432$ \\
\hline & El Sol (S)/abril & Urrao & & -76.1077 & 6.4815 & $2600-3650$ \\
\hline & $\begin{array}{l}\text { Cerro Plateado }(\mathrm{CP}) / \\
\text { agosto }\end{array}$ & Salgar & & -76.0963 & 6.0034 & $2540-3750$ \\
\hline \multirow{5}{*}{ Sonsón (SO) } & Las Palomas (P)/abril & \multirow{4}{*}{ Sonsón } & & -75.2488 & 5.7257 & $2700-3360$ \\
\hline & Norí $(\mathrm{N}) /$ mayo & & & -75.2685 & 5.8128 & $2700-3089$ \\
\hline & La Vieja (LV)/junio & & & -75.2326 & 5.774 & $2400-3300$ \\
\hline & Chaverras (Ch)/julio & & & -75.2465 & 5.6962 & $2740-2982$ \\
\hline & Valle Alto (VA)/ mayo & Salamina & Caldas & -75.2774 & 5.3527 & $3300-3485$ \\
\hline
\end{tabular}

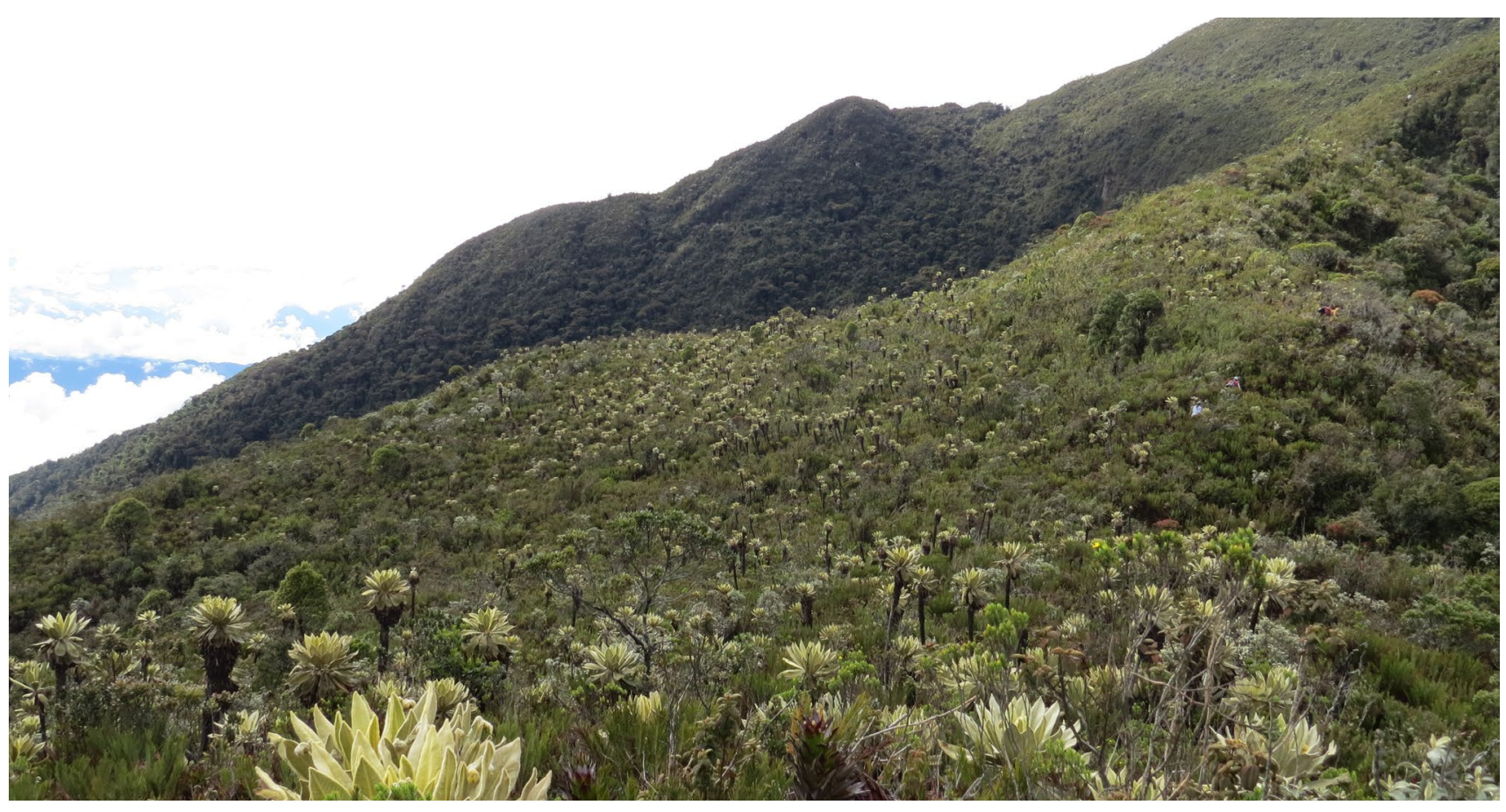

Figura 1. Cerro Plateado, municipio de Salgar, Antioquia, Colombia, perteneciente al complejo Frontino-Urrao (3700 m s. n. m.). Fotografía: Sergio Chaparro-Herrera. 


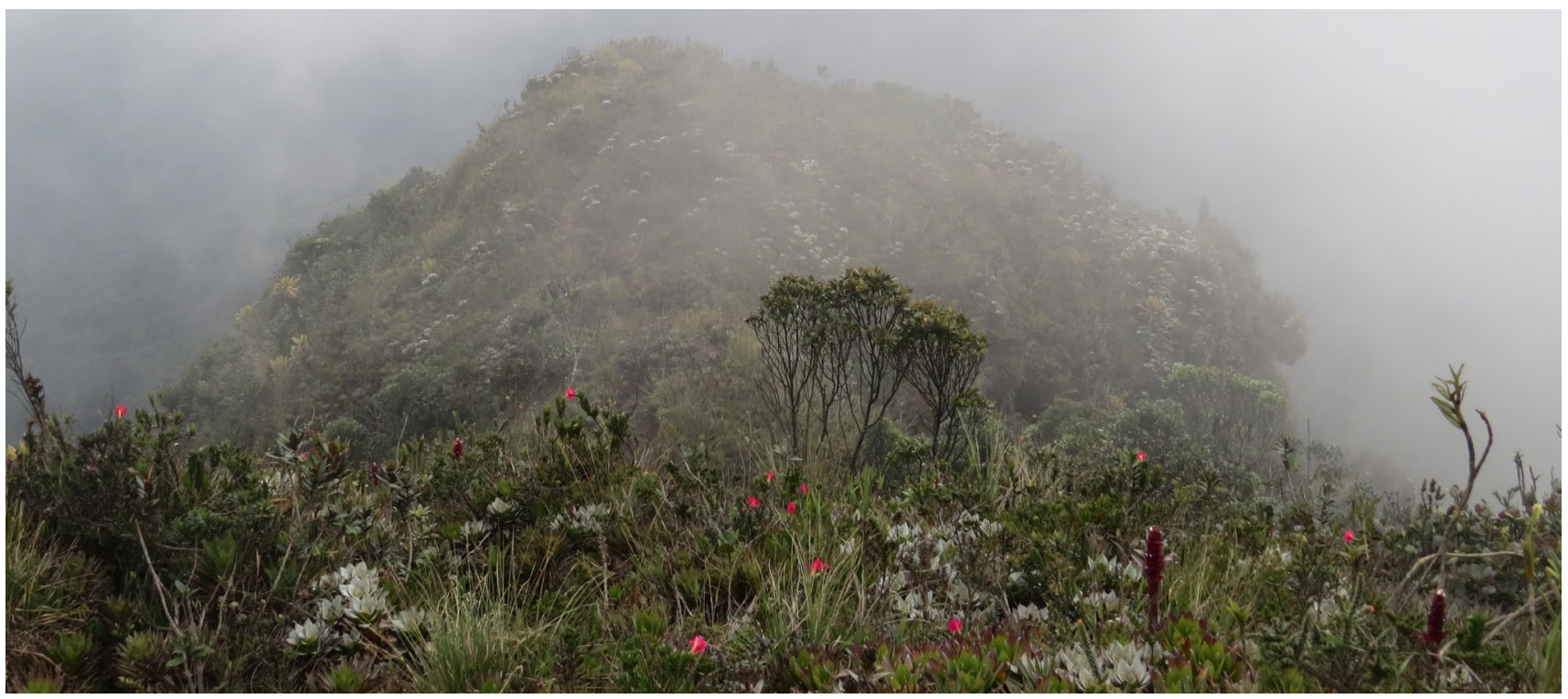

Figura 2. Las Palomas, municipio de Sonsón, Antioquia, Colombia, perteneciente al complejo Sonsón (3360 m s. n. m.). Fotografía: Sergio Chaparro-Herrera.



Figura 3. Ubicación de las localidades visitadas en los complejos de páramo Urrao-Frontino (izquierda) y Sonsón (derecha), Colombia, entre marzo y agosto de 2014. 
Registro de avifauna. Para determinar la composición de la avifauna se realizaron visitas de cinco días a cada una de las localidades entre marzo y agosto de 2014 (Tabla 1), en las que se emplearon dos métodos: observación directa en puntos de conteo y captura con redes de niebla. Además, se registraron especies observadas y escuchadas durante recorridos libres ocasionales. Para el primer método se definieron cinco estaciones de muestreo separadas por un mínimo de 50 metros en elevación, para cubrir de manera representativa el gradiente de la transición de bosque nublado hacia páramo. En cada una de las zonas de muestreo las coberturas vegetales se identificaron como bosque, transición o páramo. En cada estación se estableció un punto de conteo, que se repitió dos veces por día (entre las 6:00 y las 10:00 horas). En cada punto de conteo se hicieron registros visuales y auditivos de las especies durante 20 minutos. Para cada registro se anotaba la especie, sexo, actividad (por ej. forrajeo, vuelo, percha) y distancia de la observación. Los puntos de conteo fueron recorridos por dos observadores a distintas horas en la mañana, iniciando en extremos opuestos, alternando los puntos de inicio cada día; de esta manera se realizaron diez conteos para cada estación en cada localidad. Dado que algunas especies eran difíciles de observar, se realizaron grabaciones de las vocalizaciones utilizando una grabadora ZOOM H4n para su posterior identificación mediante comparación con plataformas especializadas. Para la segunda metodología se instalaron redes de niebla de 12 × $2 \mathrm{~m} \mathrm{(30} \mathrm{mm}$ de ojo de malla) en las estaciones donde el terreno era adecuado. El esfuerzo de muestreo se calculó en horas-red, donde una hora-red equivale a una red abierta durante una hora (Villareal et al., 2006). Para cada ave capturada se obtuvo el peso (masa corporal) con balanzas digitales con precisión de $0.1 \mathrm{~g}$, y se tomaron medidas con un calibrador con precisión de $0.1 \mathrm{~mm}$ o reglas con tope perpendicular (en el caso de las rectrices o ala). Se realizó un diminuto corte de la parte terminal de una de las plumas externas de la cola para diferenciar individuos ya capturados. Se recolectaron máximo dos individuos de especies que actualmente no estuvieran representadas $(\leq 1$ individuo) en las colecciones más importantes del país.
Estos especimenes fueron depositados en la colección del Museo de la Universidad de Antioquia. Todas las aves capturadas fueron medidas y fotografiadas (ver Montoya et al., 2018). Utilizamos las guías de Hilty \& Brown (1986) y Restall et al. (2007) para la determinación de los registros visuales y la guía sonora de Álvarez et al. (2007), la base de datos de Xenocanto (http:/ / www.xeno-canto.org/) y la guía sonora de aves de Colombia de Boesman (2012) para los registros auditivos. Las especies fueron categorizadas según el estado de amenaza nacional (Renjifo et al., 2014), endemismo (Chaparro-Herrera et al., 2013) y migración (Naranjo et al., 2012). La taxonomía siguió a Remsen et al. (http://www.museum.lsu.edu/ Remsen/SACCBaseline.htm).

\section{Resultados}

Se registraron 197 especies, pertenecientes a 40 familias. Cinco de las especies registradas son endémicas (E) de Colombia: Odontophorus hyperythrus, Coeligena orina, Scytalopus canus, Diglossa gloriosissima e Hypopyrrhus pyrohypogaster; 15 son casi endémicas (CE), 2 de interés (EI) (presentan entre el 40-49 \% de su área de distribución en Colombia), 4 migratorias boreales y 1 migratoria austral, con poblaciones reproductivas en Colombia (Tyrannus savana). Siete especies presentan alguna categoría de amenaza y una está Casi Amenazada (NT) (Anexo 1). La familia más representativa fue Thraupidae, con 29 y 27 especies en Sonsón y Frontino-Urrao, respectivamente, seguida por Tyrannidae, con 19 y 23, y Trochilidae con 21 y 16 (Figura 4). Las familias restantes presentan números de especies similares, a excepción de Accipitridae, con siete especies en el complejo Frontino-Urrao y dos en el complejo Sonsón, y Emberizidae, con cuatro especies y ocho especies, respectivamente. Finalmente, cuatro familias solo se registraron en el complejo Frontino-Urrao, representadas en todos los casos por una especie (Tinamidae, Ardeidae, Mimidae y Cardinalidae) y otras cuatro solo se registraron en el complejo de Sonsón (Cracidae, Odontophoridae, Formicariidae y Tityridae). 




Familias

Figura 4. Familias de aves más diversas en los complejos de páramo Frontino-Urrao y Sonsón, Colombia.

Complejo Frontino-Urrao: se registraron 157 especies de 36 familias (Tabla 2). En 922 horas-red, se capturaron 194 individuos pertenecientes a 48 especies y 11 familias. La localidad con mayor éxito de captura fue El Junco, con 82 individuos (33 especies). Las especies con mayor número de capturas fueron Eriocnemis vestita, Diglossa gloriosissima y Spinus spinescens con 62, 10 y 9 individuos, respectivamente. Estas especies están asociadas principalmente a páramo y transición. De estas capturas, se resalta Diglossa gloriosissima (10 individuos), capturada en todas las localidades del complejo, y Coeligena orina (1 individuo), capturada en El Junco.

Se destaca la presencia en este complejo de Coeligena orina, Scytalopus canus y Diglossa gloriosissima, especies endémicas y en peligro de extinción (EN) e Hypopyrrhus pyrohypogaster, especie endémica y vulnerable (VU) (Tabla 2, Anexo 1). A partir de la información obtenida de los censos por puntos de conteo (25 puntos) se registraron 85 especies distribuidas en 26 familias, siendo Cerro Plateado el de mayor riqueza, con 45 especies, y Pená el de menor, con 27. 
Tabla 2. Riqueza, endemismo y amenaza de las aves en los complejos de páramo Frontino-Urrao y Sonsón, Colombia.

\begin{tabular}{|c|c|c|c|c|c|c|c|}
\hline Complejo & Localidad & $\begin{array}{c}\text { Número } \\
\text { de especies } \\
\text { (exclusivas) }\end{array}$ & Endémicas & $\begin{array}{c}\text { Casi } \\
\text { endémicas }\end{array}$ & $\begin{array}{c}\text { De } \\
\text { Interés }\end{array}$ & Amenazadas & Migratorias \\
\hline \multirow{5}{*}{$\begin{array}{l}\text { Frontino } \\
\text {-Urrao }\end{array}$} & Pená & $51(4)$ & 2 & 4 & 1 & 2 (EN), 1 (NT) & 0 \\
\hline & El Junco & $80(10)$ & 3 & 5 & 0 & $2(\mathrm{EN}), 1$ (VU) & 3 \\
\hline & El Sol & $93(13)$ & 2 & 13 & 1 & $\begin{array}{c}\text { (EN), } 1(\mathrm{VU}) \\
1(\mathrm{NT})\end{array}$ & 2 \\
\hline & La Horqueta & $83(10)$ & 3 & 3 & 0 & $3(\mathrm{EN})$ & 0 \\
\hline & $\begin{array}{l}\text { Cerro } \\
\text { Plateado }\end{array}$ & $97(12)$ & 4 & 9 & 0 & $\begin{array}{c}3(\mathrm{EN}), 1(\mathrm{VU}) \\
1(\mathrm{NT})\end{array}$ & 0 \\
\hline \multirow{5}{*}{ Sonsón } & Las Palomas & $81(5)$ & 0 & 7 & 0 & $1(\mathrm{NT})$ & 1 \\
\hline & Norí & $73(11)$ & 1 & 9 & 0 & $2(\mathrm{NT})$ & 0 \\
\hline & Valle Alto & $56(11)$ & 0 & 6 & 3 & 1 (VU) & 0 \\
\hline & La Vieja & $107(25)$ & 0 & 11 & 0 & $1(\mathrm{NT})$ & 0 \\
\hline & Chaverras & $70(2)$ & 0 & 6 & 0 & 0 & 0 \\
\hline
\end{tabular}

Complejo Sonsón: se registraron 155 especies de 36 familias (Tabla 2). Con un esfuerzo de muestreo de 955.35 horas-red se capturarons 143 individuos pertenecientes a 39 especies y 11 familias. La localidad con mayor éxito de captura fue Cerro Norí con 47 individuos (17 especies). Las especies con mayor número de capturas fueron Coeligena torquata, Heliangelus exortis y Eriocnemis vestita, con 18,13 y 12 individuos, respectivamente. Estas especies se encontraron principalmente asociadas a bosque, a excepción de E. vestita, presente en páramo y transición. De estas capturas se resalta Iridosornis porphyrocephalus (un individuo) en La Vieja por ser una especie casi endémica y Casi Amenazada (NT).

Se resalta la presencia de Odontophorus hyperythrus, una especie endémica y NT (única especie endémica en el complejo, registrada en Norí) y 15 especies casi endémicas de las cuales una es Casi Amenazada (Andigena nigrirostris) (Tabla 4, Anexo 1). Finalmente, resaltamos el hallazgo de un nido activo del semillero paramuno (Catamenia homochroa) en Las Palomas, al ser la primera descripción de nido y huevos para esta especie (Chaparro-Herrera et al., 2015). A partir de la información obtenida de los censos por puntos de conteo (25 puntos) se registraron en el complejo 108 especies distribuidas en 27 familias, siendo La Vieja el de mayor riqueza con 55 especies y Valle Alto el de menor con 37 especies.

Especies de importancia. A continuación se resaltan algunas especies registradas en los dos complejos de páramo ya sea por su grado de amenaza nacional o por presentar ampliación en el rango de distribución y altitudinal:

Odontophorus hyperythrus. Endémica, categorizada nacionalmente como casi amenazada (NT) hasta 2013 y reevaluada en preocupación menor (LC) en la actualidad (Chaparro-Herrera et al., 2013; Renjifo et al., 2002; Renjifo et al., 2014). Presente en la cordillera Occidental y Central y cabecera del valle del Magdalena en Huila en ambas vertientes entre los 1600 y los $2800 \mathrm{~m} \mathrm{~s}$. n. m. (Hilty \& Brown, 1986; Carroll et al., 2016). Registrada en este estudio auditivamente en Norí a los 2975 m s. n. m. asociada a transición entre el páramo y el bosque.

Hapalopsittaca amazonina. Casi endémica y vulnerable (VU) (Chaparro-Herrera et al., 2013; Renjifo et al., 2014). Se ha reportado entre los 2000 y 3600 m s. n. m. en la vertiente occidental de la cordillera Oriental y en el norte de la vertiente oriental de la cordillera Occiden- 
tal y en ambas laderas de la cordillera Central (Hilty \& Brown, 1986; Sanabria-Mejía \& Mayorquín-Cabrera, 2014; Collar \& Boesman, 2016). Fue registrada en este estudio visual y auditivamente en grupos de entre tres y cinco individuos que sobrevolaban en varias ocasiones El Sol (3650 m s. n. m.), siendo un área de importancia para la conservación de esta especie, al ser la única localidad conocida al norte de los Andes occidentales.

Andigena nigrirostris. Casi endémica y casi amenazada (NT) (Chaparro-Herrera et al., 2013; Renjifo et al., 2014). Se encuentra a lo largo de los Andes entre los 1600 y los 3245 m s. n. m., principalmente (rara vez a los $1200 \mathrm{~m}$ s. n. m.) (Garcés-Restrepo, 2014; Short, 2016). Registrada visual y auditivamente en este estudio en Pená, El Sol, Cerro Plateado en el complejo de Frontino-Urrao (A. n. occidentalis) y Las Palomas, Norí y La Vieja en el complejo de Sonsón (A. n. spilorhynchus), asociada a transición entre bosque y páramos. Resaltamos la presencia de A. n. occidentalis en Pená y El Sol a los 3373 y $3541 \mathrm{~m}$ s. n. m., respectivamente, al ser las elevaciones más altas conocidas para la especie.

Andigena hypoglauca. Vulnerable (VU) (Renjifo et al., 2014). Se encuentra entre los 2700 y los 3400 m s. n. m. a lo largo de la cordillera Central, desde el PNN Los Nevados hacia el sur hasta el PNN Puracé, y en el Macizo Colombiano en la vertiente oriental en Nariño y posiblemente en Putumayo (Garcés-Restrepo \& Renjifo, 2014; Short \& Kirwan, 2016). En este estudio fue registrada y fotografiada una pareja en Valle Alto a los 3475 m s. n. m., consumiendo frutos en arbustos en cercanías a relictos de bosque. Este registro amplía $30 \mathrm{~km}$ aproximadamente la distribución hacia el norte de la cordillera Central.

Coeligena orina. Endémica y en peligro (EN) (Chaparro-Herrera et al., 2013; Renjifo et al., 2014). Ha sido registrada en varias localidades en la cordillera Occidental entre los 3100 y $3500 \mathrm{~m}$ s. n. m., principalmente (a los 2500 Cerro Montezuma) (Flórez et al., 2004; Krabbe et al., 2005; Pulgarín-R. \& Múnera-P, 2006; López-Ordoñez et al., 2013; Carantón \& Suárez, 2014). Se presume de su posible ocurrencia en páramos de Paramillo (Antioquia), Serranía de los Paraguas (Chocó), Farallones de
Cali (Valle del Cauca), Páramo del Duende (Valle del Cauca-Chocó) y posiblemente en el páramo de Argelia-Serranía del Pinche y Munchique (Cauca) (Krabbe et al., 2005; López-Ordoñez et al., 2013). En este estudio fue registrada visualmente en todas las localidades del complejo de Frontino-Urrao a excepción de Pená, pero se presume que se encuentre también allí, por las características de la vegetación y por la cercanía a El Junco, donde fue capturado un individuo. Todos los registros fueron realizados en el bosque en altitudes que van entre los 3437 y 3540 m s. n. m., ampliando a diez el número de localidades conocidas para la especie.

Pterophanes cyanopterus. distribuida en la cordillera Central, desde Caldas en el sur hasta Nariño y en la cordillera Oriental desde Norte de Santander hasta Cundinamarca entre los 2800 y $3800 \mathrm{~m} \mathrm{~s}$. n. m. (usualmente por encima de los 3000 m s. n. m.) (Hilty \& Brown, 1986; Verhelst, 2001; Ayerbe-Quiñones et al., 2015; Heynen et al., 2016). En este estudio fue registrado (P. c. caeruleus) visualmente en Las Palomas y La Vieja, complejo de Sonsón, a los 3363 y 3247 m s. n. m., respetivamente, asociados a páramo, siendo los primeros registros para el departamento de Antioquia y las localidades más al norte en la cordillera Central (Chaparro-Herrera et al., 2017).

Grallaria alleni. Casi endémica y en peligro (EN) (Chaparro-Herrera et al., 2013; Renjifo et al., 2014). Se distribuye entre los 1850 y los $3490 \mathrm{~m}$ s. n. m., en la vertiente occidental de la cordillera Central, alto Magdalena y descubierta recientemente en el páramo de Frontino, Apía y Cerro Montezuma (Krabbe et al., 2005; López-Ordóñez et al., 2013; Ocampo-Peñuela et al., 2015). Fue registrada en este estudio auditivamente en El Sol a los 3490 m s. n. m., asociada al interior de bosque, siendo este un nuevo registro para esta localidad y el registro de mayor elevación reportado hasta el momento.

Scytalopus canus. Endémica y EN (Chaparro-Herrera et al., 2013; Renjifo et al., 2014). Se encuentra entre los 3000 y 3500 m s. n. m. (3800 en Paramillo), al norte de la cordillera Occidental, posiblemente en los Farallones de Citará (Krabbe \& Cadena, 2010; Navas-Berdugo \& Cadena, 2014). Asimismo, fue registrada auditivamente en el 
PNN Tatamá, ampliando su distribución considerablemente hacia el sur (ver: www.xeno-canto.org/145482). En este estudio fue registrada visual y auditivamente entre los 3374 y los 3750 m s. n. m. en Pená, El Junco, La Horqueta y Cerro Plateado, asociada a páramo, transición y bosque, siendo este último el de mayor presencia. Los registros presentados aumentan el número de localidades conocidas para la especie, resaltando Cerro Plateado (http:/ / www.xeno-canto.org/321599), que se encuentra a una distancia de $43 \mathrm{~km}$ del páramo de Frontino y $25 \mathrm{~km}$ de Farallones de Citará, posible lugar de presencia de la especie, lo que podría indicar su presencia continua en las zonas altas de la cordillera Occidental entre Paramillo y PNN Tatamá.

Ampelion rufaxilla. Vulnerable (VU) (Renjifo et al., 2014); se encuentra en ambas vertientes de las cordilleras Occidental y Central, en donde existen registros de pocas localidades entre los 1750 y los 3374 m s. n. m. (más común por encima de los $2300 \mathrm{~m}$ s. n. m., una vez a 3100 en el páramo de Frontino) (Krabbe et al., 2005; Castaño-Hernández \& Ocampo-Peñuela, 2014; Snow, 2016). Registrada en este estudio visualmente en El Junco, a los $3374 \mathrm{~m}$ s. n. m. en interior de bosque, siendo una nueva localidad conocida para la especie y la de mayor elevación.

Iridosornis porphyrocephalus. Casi endémica, categorizada nacionalmente como casi amenazada (NT) hasta 2013 y reevaluada en preocupación menor (LC) en la actualidad (Chaparro-Herrera et al., 2013; Renjifo et al., 2002; Renjifo et al., 2014). Se encuentra distribuida entre 750 y 2900 m s. n. m. Fue registrada visualmente en Norí, Chaverras y La Vieja; un individuo capturado en interior de bosque en esta última localidad a $2900 \mathrm{~m} \mathrm{~s}$. n. m., siendo la localidad a mayor elevación conocida para la especie.

Diglossa gloriosissima. Endémica y en peligro (EN) (Chaparro-Herrera et al., 2013; Renjifo et al., 2014). Se encuentra distribuida entre los 2400 y 3800 m s. n. m. (principalmente entre los 3000-3800), en el Nudo de Paramillo (Ituango), páramo de Frontino (Urrao), Reserva Mesenia-Paramillo (Jardín), Farallones de Citará
(Antioquia-Chocó), PNN Tatamá (Risaralda-Chocó) y Serranía del Pinche (Argelia-Cauca) (Flórez et al., 2004; Pulgarín-R \& Múnera-P, 2006; Carantón, 2014; Ocampo-Peñuela \& Pimm, 2015; Hilty, 2016). En este estudio fue una especie común entre los 3350 y los $3750 \mathrm{~m} \mathrm{~s}$. n. m., registrada visual y auditivamente en todas las localidades, asociada a páramos, bosques y principalmente a zonas de transición entre estos dos. Fue capturada en Pená ( $n=1)$, El Junco $(n=5)$, El Sol $(n=2)$, La Horqueta $(n=1)$ y Cerro Plateado $(n=1)$.

Hypopyrrhus pyrohypogaster. Endémica y vulnerable (VU) (Chaparro-Herrera et al., 2013; Renjifo et al., 2014). Presenta una distribución restringida en el norte de las cordilleras Central y Occidental y el sector sur de la cordillera Oriental entre 800 y 2750 m s. n. m., principalmente entre 1200 y 2400 m (Garizábal et al., 2014; Callejas \& Chaparro-Herrera, 2018). En este estudio fue registrado visual y auditivamente y fotografiada en La Horqueta, a 2100 m s. n. m., en borde de bosque y en Cerro Plateado, a los 2740 m s. n. m., asociada a vegetación riparia.

Finalmente, resaltamos las siguientes especies de interés (EI) debido a que presentan entre el 40-49 \% de su área de distribución en Colombia y cuya categoría busca resaltar la elevada proporción de distribución de la especie en el país y la responsabilidad de su conservación (Chaparro-Herrera et al., 2013): Metallura williami (subespecie M. w. recisa observada en Pená complejo Frontino-Urrao y M. w. williami registrada en Valle Alto complejo Sonsón), Piranga rubriceps (registrada en El Sol, complejo Frontino-Urrao), y Chalcostigma herrani herrani y Coeligena lutetiae lutetiae (registradas en Valle Alto, complejo Sonsón).

\section{Discusión}

A pesar de que el muestreo en los complejos estudiados puede considerarse representativo, es recomendable tener una mayor cobertura en términos de las épocas del año muestreadas. Además, en algunos hábitats donde 
las condiciones de visibilidad no eran óptimas por la constante neblina propia de estos ambientes, la detección de ciertas especies era más difícil, al igual que en sotobosques muy densos. Esto, sumado a los pocos días disponibles para muestreo en relación con el tamaño del área, probablemente afectó el número de registros, tanto de individuos como de especies.

Los ecosistemas de alta montaña ofrecen importantes servicios ecosistémicos, como la regulación del ciclo hídrico y retención de carbono, entre otros, además de ser áreas de importancia para la conservación al albergar una gran riqueza de especies endémicas (Morales et al., 2007; Sarmiento et al., 2013; Cabrera \& Ramírez, 2014; Marín \& Parra, 2015; Suárez-Sanabria \& Cadena, 2014). Sin embargo son pocos los estudios de aves realizados en áreas de páramo (por ej., Krabbe et al., 2005; Pulgarín-R \& Múnera-P, 2006; Salamanca-Reyes, 2011; Suárez-Sanabria \& Cadena, 2014), y, por lo tanto, existen grandes vacíos de información respecto a la presencia y distribución de la avifauna en estos ecosistemas. Por otro lado, hasta la fecha solo se conocía una exploración realizada en el páramo de El Sol en un gradiente de elevación más amplio, 2500-3900 m s. n. m., y con mayor esfuerzo de muestreo (Flórez et al., 2004; Krabbe et al., 2005) en la que se registraron 155 especies, una cifra cercana a la registrada en todo el complejo Frontino-Urrao. Sin embargo, la avifauna registrada en este estudio es similar a la reportada por Krabbe et al. (2005), con algunas diferencias en el número de especies por familia asociadas a ambientes boscosos, como Furnariidae y Thraupidae.

El hallazgo de Coeligena orina y Diglossa gloriossisima en la mayoría de los páramos visitados podría evidenciar conectividad entre ellos, que es clave para la persistencia de especies endémicas amenazadas (ver Renjifo et al., 2014). Pulgarín-R \& Múnera-P (2006) consideraron "común" a Coeligena orina en los Farallones de Citará, asociada principalmente a bosque enano, hierba paramuna y en la parte media del bosque. Flórez et al. (2004) reportaron la especie en el páramo de Frontino (denominado aquí El Sol) como "poco común" y asociada al ecotono entre páramo-bosque ena- no y bosques húmedo alto entre 3150 y $3500 \mathrm{~m} \mathrm{~s}$. n. $\mathrm{m}$. Por su parte, Diglossa gloriosissima fue una especie común en nuestro estudio, asociada principalmente a zonas de transición. Flórez et al. (2004) mencionan que la especie es común en el páramo de Frontino entre 3100 y $3600 \mathrm{~m}$ s. n. m. y se asocia a parches de bosque achaparrado, mientras que Pulgarín-R. \& Múnera-P. (2006) reportaron en los Farallones de Citará cinco individuos asociados principalmente a bosque enano y vegetación paramuna. Esto evidencia la preferencia por hábitats como páramo y zonas de transición. A pesar de la importancia de los ecosistemas en la cordillera Occidental en términos de endemismo y aves amenazadas (Velásquez-Tibatá et al., 2013), son muy pocas las áreas protegidas que incluyen ecosistemas de páramo, y la pequeña extensión de estos ecosistemas enfatiza la vulnerabilidad ante cambios en el uso de la tierra y cambio climático (Silva-Muñoz, 2015).

En el complejo de Sonsón se registraron 55 especies de aves más que en en los páramos de Los Nevados, entre 3414 y $4026 \mathrm{~m} \mathrm{~s}$. n. m., $8 \mathrm{~km}$ lineales al sur sobre la misma cordillera Central. Sin embargo, en esa zona se registraron 15 especies no halladas en Sonsón (http:/ / doi.org/10.15472/rs81k8). Por su parte, en el complejo Las Hermosas y Chilí-Barragán, situado 96 km lineales al sur de Sonsón, entre 3100 y 3520 m s. n. m., se registraron 25 especies menos que en Sonsón; no obstante, ese estudio incluyó 29 especies diferentes (http:/ / doi. org/10.15472/jsb352).

Entre los registros no presentes en el complejo de Sonsón se cuentan especies cuya distribución en la cordillera Central termina, de sur a norte, justo en las estribaciones del Nevado del Ruiz (Hilty \& Brown, 1986). Graham et al. (2010) identificaron esta zona como una discontinuidad ambiental y la caracterizan como una transición de Sur a Norte de condiciones frías a cálidas y ligeramente más secas, lo cual podría explicar la ausencia de estas especies en el complejo Sonsón. Sin embargo, algunas de ellas se podrían encontrar más allá de esta discontinuidad ambiental, como es el caso de Pterophanes cyanopterus, registrada en Las Palomas y La Vieja, donde es una especie rara. Su distribución 
coincide con este "límite" pues el área disponible en elevaciones por encima de los $3000 \mathrm{~m} \mathrm{~s}$. n. m. disminuye de manera considerable alrededor del límite entre Caldas y Antioquia, reduciendo así el área potencial para la especie hacia el norte de la cordillera Central (Chaparro-Herrera et al., 2017).

\section{Conclusiones}

Los pocos estudios hechos en estas localidades hacen prioritario el levantamiento de información primaria de estas zonas con alto grado de endemismo, y que permita una comparación apropiada de la representatividad de la avifauna registrada en este trabajo, así como la identificación de amenazas que enfrentan las especies a lo largo de su distribución de manera local. Esperamos que los registros presentados en este trabajo sirvan de base para la toma de decisiones de conservación.

\section{Agradecimientos}

A Paola Montoya Valencia por la toma de datos en campo, así como la revisión inicial del manuscrito; al Programa Fondo Adaptación del Ministerio de Ambiente y Desarrollo Sostenible de Colombia, al Instituto de Investigación de Recursos Biológicos Alexander von Humboldt y a la Universidad de Antioquia, por la financiación y ejecución del proyecto. A los asistentes de campo: Laura Franco, Héctor Manuel Arango, Michael Castaño, Edwin Hurtado, Natalia Yepes y Sergio Montoya, por el apoyo en la toma de algunos datos.

\section{Referencias}

Álvarez, M., Caro, V., Laverde, O. \& Cuervo, A. M. (2007). Guía sonora de las aves de los Andes Colombianos. Bogotá: Instituto Alexander von Humboldt. Ayerbe-Quiñones, F. (2015). Colibríes de Colombia. Serie: Avifauna Colombiana. Bogotá: Wildlife Conservancy Society. 352 pp.
Boesman, P. (2012). Birds of Colombia - Mp3 Sound Collection (1.0). Merelbeke, Belgium.

Cabrera, M. \& Ramírez, W. (Eds). (2014). Restauración ecológica de los páramos de Colombia. Transformación y herramientas para su conservación. Bogotá: Instituto de Investigación de Recursos Biológicos Alexander von Humboldt (IAvH). 296 pp.

Callejas, D. \& Chaparro-Herrera, S. (2018). Registro del Chango Colombiano Hypopyrrhus pyrohypogaster en el departamento del Meta, Colombia. Cotinga, 40, 95-97.

Carantón, D. (2014). Diglossa gloriosissima. En Renjifo, L. M., Gómez, M. F., Velásquez-Tibatá, J., Amaya-Villarreal, A. M., Kattan, G. H., Amaya-Espinel, J. D. \& Burbano-Girón, J (Eds.). Libro rojo de aves de Colombia, Volumen I: bosques húmedos de los Andes y la costa Pacífica. Pp. 319-321. Bogotá: Editorial Pontificia Universidad Javeriana e Instituto Alexander von Humboldt.

Carantón, D. \& Suárez, G. (2014). Coeligena orina. En Renjifo, L. M., Gómez, M. F., Velásquez-Tibatá, J., Amaya-Villarreal, A. M., Kattan, G. H., Amaya-Espinel, J. D. \& Burbano-Girón, J (Eds.). Libro rojo de aves de Colombia, Volumen I: bosques húmedos de los Andes y la costa Pacífica. Pp. 134-136. Bogotá: Editorial Pontificia Universidad Javeriana e Instituto Alexander von Humboldt.

Carroll, J. P., Kirwan, G. M. \& Sharpe, C. J. (2016). Chestnut Wood-quail (Odontophorus hyperythrus). En del Hoyo, J., Elliott, A., Sargatal, J., Christie, D. A. \& de Juana, E (Eds.). Handbook of the Birds of the World Alive. Barcelona: Lynx Edicions. Recuperado el 29 de junio de 2016 de http:/ / www.hbw.com/ node/53355.

Castaño-Hernández, J. \& Ocampo-Peñuela, N. (2014). Ampelion rufaxilla. En Renjifo, L. M., Gómez, M. F., Velásquez-Tibatá, J., Amaya-Villarreal, A. M., Kattan, G. H., Amaya-Espinel, J. D. \& Burbano-Girón, J (Eds.). Libro rojo de aves de Colombia, Volumen I: bosques húmedos de los Andes y la costa Pacífica. Pp. 252254. Bogotá: Editorial Pontificia Universidad Javeriana e Instituto Alexander von Humboldt.

Chaparro-Herrera, S., Echeverry-Galvis, M. Á., Córdoba-Córdoba, S. \& Sua-Becerra, A. (2013). Listado actualizado de las aves endémicas y casi-endémicas de Colombia. Biota Colombiana, 14(2), 235-272. 
Chaparro-Herrera, S., Montoya, P. \& Borges, O. B. (2015). Anidación del Semillero Paramuno (Catamenia homochroa) en Colombia y Brasil. Ornitología Neotropical, 26, 295-298.

Chaparro-Herrera, S., Montoya, P., Rivera, H. \& Parra, J. L. (2017). Primeros registros del Colibrí Aliazul (Pterophanes cyanopterus) en Antioquia. Biota Colombiana, 18(2), 262-266.

Collar, N. \& Boesman, P. (2016). Rusty-faced Parrot (Hapalopsittaca amazonina). En del Hoyo, J., Elliott, A., Sargatal, J., Christie, D. A. \& de Juana, E. (Eds.) Handbook of the Birds of the World Alive. Barcelona: Lynx Edicions. Recuperado el 15 de junio de 2016 de http://www.hbw.com/node/54720.

Flórez, P., Krabbe, N., Castaño, J., Suárez, G. \& Arango, J. D. (2004). Evaluación Avifauna del Páramo de Frontino, Antioquia, Agosto 2004. (Informe técnico). Colombian EBA Project Report Series No. 6. Bogotá D. C.: Fundación ProAves. 27 pp.

Garcés-Restrepo, M. F. (2014). Andigena nigrirostris. En Renjifo, L. M., Gómez, M. F., Velásquez-Tibatá, J., Amaya-Villarreal, A. M., Kattan, G. H., Amaya-Espinel, J. D. \& Burbano-Girón, J. (Eds.). Libro rojo de aves de Colombia, Volumen I: bosques húmedos de los Andes y la costa Pacífica. Pp. 390-391. Bogotá: Editorial Pontificia Universidad Javeriana e Instituto Alexander von Humboldt.

Garcés-Restrepo, M. \& Renjifo, L. M. (2014). Andigena hypoglauca. En Renjifo, L. M., Gómez, M. F., Velásquez-Tibatá, J., Amaya-Villarreal, A. M., Kattan, G. H., Amaya-Espinel, J. D. \& Burbano-Girón, J. (Eds.). Libro rojo de aves de Colombia, Volumen I: bosques húmedos de los Andes y la costa Pacífica. Pp. 144-146. Bogotá: Editorial Pontificia Universidad Javeriana e Instituto Alexander von Humboldt.

Garizábal, J. A., Londoño, L. V. \& Cuervo, A. M. (2014). Hypopyrrhus pyrohypogaster. En Renjifo, L. M., Gómez, M. F., Velásquez-Tibatá, J., Amaya-Villarreal, A. M., Kattan, G. H., Amaya-Espinel, J. D. \& Burbano-Girón, J. (Eds.). Libro rojo de aves de Colombia, Volumen I: bosques húmedos de los Andes y la costa Pacífica. Pp. 357-360. Bogotá: Editorial Pontificia Universidad Javeriana e Instituto Alexander von Humboldt.

Graham, C., Silva, N. \& Velásquez-Tibatá, J. (2010). Evaluating the potential causes of range limits of birds of the Colombian Andes. Journal of Biogeography, 37, 1863-1875.

Heynen, I., Boesman, P. \& Kirwan, G. M. (2016). Great Sapphirewing (Pterophanes cyanopterus). En del Hoyo, J., Elliott, A., Sargatal, J., Christie, D. A. \& de Juana, E. (Eds.). Handbook of the Birds of the World Alive. Barcelona: Lynx Edicions. Recuperado el 20 de junio de 2016 de http:/ / www.hbw.com/ node/55571.

Hilty, S. L. \& Brown, W. L. (1986). A guide to the birds of Colombia. New Jersey: Princeton University Press. 836 pp. Hilty, S. (2016). Chestnut-bellied Flowerpiercer (Diglossa gloriosissima). En del Hoyo, J., Elliott, A., Sargatal, J., Christie, D. A. \& de Juana, E. (Eds.). Handbook of the Birds of the World Alive. Barcelona: Lynx Edicions. Recuperado el 15 de junio de 2016 de http:/ / www. hbw.com/node/61764.

Hilty, S. (2017). Flame-rumped Tanager (Ramphocelus flammigerus). En del Hoyo, J., Elliott, A., Sargatal, J., Christie, D. A. \& de Juana, E. (Eds.). Handbook of the Birds of the World Alive. Barcelona: Lynx Edicions. Recuperado el 4 de septiembre de 2017 de http:/ / www.hbw.com/node/61632.

Krabbe, N. \& Cadena, C. D. (2010). A taxonomic revision of the Páramo Tapaculo Scytalopus canus Chapman (Aves: Rhinocryptidae), with description of a new subspecies from Ecuador and Peru. Zootaxa, 2354, 56-66.

Krabbe, N., Flórez, P., Suárez, G., Castaño, J., Arango, J. D., Pulgarín, P. C., Múnera, W., Stiles, F. G. \& Salaman, P. (2005). Rediscovery of the Dusky Starfrontlet Coeligena orina, with a description of the adult plumages and a reassessment of its taxonomic status. Ornitología Colombiana, 3, 28-35.

López-Ordóñez, J. P., Cortés-Herrera, J. O., Paez-Ortíz, C. A. \& González-Rojas, M. F. (2013). Nuevos registros y comentarios sobre la distribución de algunas especies de aves en los Andes Occidentales de Colombia. Ornitología Colombiana, 13, 21-36.

Marín, C. \& Parra, S. (2015). Bitácora de flora: Guía visual de plantas de páramos en Colombia. Bogotá: Instituto de Investigación de Recursos Biológicos Alexander von Humboldt. 356 pp.

Montoya, P. M., González, A., Tenorio, E. A., López-Ordóñez, J. P., Pinto, A., Cueva, D., Acevedo Rincón, 
A. A., Angarita Yanes, C. , Arango Martínez, H. M., Armesto, O., Betancur, J. S., Caguazango Castro, A. , Calderon Leyton, J. J., Calpa-Anaguano, E. V., Cárdenas-Posada, G. , Castaño Díaz, M. , Chaparro-Herrera, S. , Diago-Muñoz, N. , Franco Espinosa, L. , Gómez Bernal, L. G., Gonzalez-Zapata, F. L., Gutiérrez Zamora, E. A., Gutiérrez-Zuluaga, A. M., Lizcano Jiménez, R. S., Lopera-Salazar, A. , Alvarado, D. M., Maya Girón, A. M., Medina, W. , Montealegre-Talero, C. , Parra, J. L., Pérez-Peña, S. , Ramírez Ramírez, F. , Reyes, J. , Rivera-Gutiérrez, H. F., Rosero Mora, Y. , Trujillo-Torres, C. M., Vidal-Maldonado, C. C. \& Salgado-Negret, B. (2018). A morphological database for 606 Colombian bird species. Ecology, doi:10.1002/ecy.2368.

Morales, M., Otero, J., Van der Hammen, T., Torres, A., Cadena, C., Pedraza, C., Rodríguez, N., Franco, C., Betancourth, J. C., Olaya, E., Posada, E. \& Cárdenas, L. (2007). Atlas de páramos de Colombia. Bogotá: Instituto de Investigación de Recursos Biológicos Alexander von Humboldt. 208 pp.

Morales, M. \& Armenteras, D. (2013). Estado de conservación de los bosques de niebla de los Andes colombianos, un análisis multiescalar. Boletín Científico Museo de Historia Natural, 17(1), 64-72.

Mulligan, M. \& Burke, S. M. (2005). DFID FRP Project ZF0216 Global cloud forests and environmental change in a hydrological context. (Informe técnico). London: King's College. 74 pp.

Naranjo, L. G., Amaya, J. D., Eusse-González, D. \& Cifuentes-Sarmiento, Y. (Eds.). (2012). Guía de las Especies Migratorias de la Biodiversidad en Colombia. Aves. Vol. 1. Bogotá: Ministerio de Ambiente y Desarrollo Sostenible/ WWF Colombia. 708 pp.

Navas-Berdugo, A. P. \& Cadena, C. D. (2014). Scytalopus canus. En Renjifo, L. M., Gómez, M. F., Velásquez-Tibatá, J., Amaya-Villarreal, A. M., Kattan, G. H., Amaya-Espinel, J. D. \& Burbano-Girón, J. (Eds.). Libro rojo de aves de Colombia, Volumen I: bosques húmedos de los Andes y la costa Pacífica. Pp. 240-242. Bogotá: Editorial Pontificia Universidad Javeriana e Instituto Alexander von Humboldt.

Ocampo-Peñuela, N. F \& Pimm, S. L. (2015). Elevational ranges of montane birds and deforestation in the Western Andes of Colombia. PLoS ONE, 10(12), e0143311.
Pulgarín-R., P.C. \& Múnera-P, W. (2006). New bird records from Farallones del Citará, Colombian Western Cordillera. SAO, 16, 44-53.

Renjifo, L. M., Franco-Maya, A. M., Amaya-Espinel, J. D., Kattan, G. H. \& López-Lanús, B. (Eds.). (2002). Libro rojo de aves de Colombia. Serie Libros Rojos de Especies Amenazadas de Colombia. Bogotá: Instituto de Investigación de Recursos Biológicos Alexander von Humboldt y Ministerio del Medio Ambiente. 562 pp.

Renjifo, L. M., Gómez, M. F., Velásquez-Tibatá, J., Amaya-Villarreal, A. M., Kattan, G. H., Amaya-Espinel, J. D. \& Burbano-Girón, J. (Eds.). (2014). Libro rojo de aves de Colombia, Volumen I: bosques húmedos de los Andes y la costa Pacífica. Bogotá: Editorial Pontificia Universidad Javeriana e Instituto Alexander von Humboldt. 465 pp.

Restall, R., Rodner, C. \& Lentino, M. (2007). Birds of Northern South America: An identification Guide: Species Accounts. New Haven: Yale University Press. 880 pp.

Rivera, D. \& Rodríguez, C. (2011). Guía divulgativa de criterios para la delimitación de páramos de Colombia. Bogotá: Ministerio de Ambiente, Vivienda y Desarrollo Territorial e Instituto de Investigación de Recursos Biológicos Alexander von Humboldt. 68 pp.

Salamanca-Reyes, J. R. (2011). Ecología del Barbudito de Páramo (Oxypogon guerinii, Trochilidae) en el páramo de Siscunsí, Boyacá, Colombia. Ornitología Colombiana, 11, 58-75.

Sanabria-Mejía, Y. \& Mayorquín-Cabrera, A. (2014). Hapalopsittaca amazonina. En Renjifo, L. M., Gómez, M. F., Velásquez-Tibatá, J., Amaya-Villarreal, A. M., Kattan, G. H., Amaya-Espinel, J. D. \& Burbano-Girón, J. (Eds.)(Eds.). Libro rojo de aves de Colombia, Volumen I: bosques húmedos de los Andes y la costa Pacífica. Pp. 187-190. Bogotá: Editorial Pontificia Universidad Javeriana e Instituto Alexander von Humboldt.

Sarmiento, C., Cadena, C., Sarmiento, M., Zapata, J. \& León, O. (2013). Aportes a la conservación estratégica de los páramos de Colombia: Actualización de la cartografía de los complejos de páramo a escala 1:100.000. Bogotá: Instituto de Investigación de Recursos Biológicos Alexander von Humboldt. 89 pp.

Short, L. L. (2016). Black-billed Mountain-toucan (Andigena nigrirostris). En del Hoyo, J., Elliott, A., Sargatal, 
J., Christie, D. A. \& de Juana, E. (Eds.). Handbook of the Birds of the World Alive. Barcelona: Lynx Edicions. Recuperado el 16 de junio de 2016 de http:/ / www. hbw.com/node/56085 el 16 junio 2016.

Short, L. L. \& Kirwan, G. M. (2016). Grey-breasted Mountain-toucan (Andigena hypoglauca En del Hoyo, J., Elliott, A., Sargatal, J., Christie, D. A. \& de Juana, E. (Eds.). Handbook of the Birds of the World Alive. Barcelona: Lynx Edicions. Recuperado el 16 de junio de 2016 de http:/ / www.hbw.com/node/56082.

Silva-Muñoz, M. (2015). Asumiendo el reto para la conservación frente al cambio climático en el PNN Las Orquídeas. (Trabajo de grado). Universidad de Antioquia, Facultad de Ciencias Exactas y Naturales, Instituto de Biología. Medellín. 33 pp.

Snow, D. (2016). Chestnut-crested Cotinga (Ampelion rufaxilla). En del Hoyo, J., Elliott, A., Sargatal, J., Christie, D. A. \& de Juana, E. (Eds.). Handbook of the Birds of the World Alive. Barcelona, Lynx Edicions. Recuperado el 16 de julio de 2016 de http:/ / www.hbw. com/node/56996.

Suárez-Sanabria, N. \& Cadena, C. D. (2014). Diversidad y estructura de la avifauna del Valle de Lagunillas, Parque Nacional Natural El Cocuy, Colombia. Ornitología Colombiana, 14, 48-61.
Velásquez-Tibatá, J., Salaman, P. \& Graham, C. H. (2013). Effects of climate change on species distribution, community structure, and conservation of birds in protected areas in Colombia. Regional Environmental Change, 13(2), 235-248.

Verhelst, J. C., Rodríguez, J. C., Orrego, O., Botero, J. E., López, J. A., Franco, V. M. \& Pfeifer, A. M. (2001). Aves del Municipio de Manizales- Caldas, Colombia. Biota Colombiana, 2(3), 265-284.

Villarreal H., Álvarez, M., Córdoba, S., Escobar, F., Fagua, G., Gast, F., Mendoza, H., Ospina, M. \& Umaña, A. M. (2006). Manual de métodos para el desarrollo de inventarios de biodiversidad segunda edición. Bogotá: Programa de Inventarios de Biodiversidad. Instituto de Investigación de Recursos Biológicos Alexander von Humboldt. 236 pp.

Weller, A. A. \& Boesman, P. (2017). Steely-vented Hummingbird (Amazilia saucerottei). En del Hoyo, J., Elliott, A., Sargatal, J., Christie, D. A. \& de Juana, E. (Eds.). Handbook of the Birds of the World Alive. Barcelona, Lynx Edicions. Recuperado el 4 de septiembre de 2017 de http://www.hbw.com/ node/55506. 
Anexo 1. Especies de aves registradas en cada uno de los páramos de los complejos Frontino-Urrao y Sonsón, entre marzo y agosto de 2014.

Disponible en línea: http://revistas.humboldt.org.co/index.php/biota/rt/suppFiles/517/0

\section{Sergio Chaparro-Herrera}

Universidad de Antioquia,

Grupo de Ecología y Evolución de Vertebrados

Medellín, Colombia

(Autor de correspondencia)

sergioupn@gmail.com

https://orcid.org/0000-0002-3434-6347

\section{Andrea Lopera-Salazar}

Universidad de Antioquia,

Grupo de Ecología y Evolución de Vertebrados

Medellín, Colombia

alopera4@gmail.com

https://orcid.org/0000-0002-4627-3907

\section{Ana M. Gutiérrez-Zuluaga}

Universidad de Antioquia,

Grupo de Ecología y Evolución de Vertebrados

Medellín, Colombia

anamaria.gzuluaga@gmail.com

https://orcid.org/0000-0001-5801-6820

\section{Jefry Betancur}

Universidad de Antioquia,

Grupo de Ecología y Evolución de Vertebrados

Medellín, Colombia

styf17@gmail.com

https://orcid.org/0000-0003-3655-4411

\section{Dariel Martínez Alvarado}

Universidad de Antioquia,

Grupo de Ecología y Evolución de Vertebrados

Medellín, Colombia

darie104@gmail.com

https://orcid.org/0000-0003-2358-2878

\section{Héctor Fabio Rivera Gutiérrez}

Universidad de Antioquia,

Grupo de Ecología y Evolución de Vertebrados

Medellín, Colombia

fabio.rivera@udea.edu.co

https://orcid.org/0000-0001-6025-3470

Juan L. Parra

Universidad de Antioquia,

Grupo de Ecología y Evolución de Vertebrados

Medellín, Colombia

juanl.parra@udea.edu.co

https://orcid.org/0000-0002-5689-1872
Avifauna en dos complejos de páramo de Antioquia, Colombia

Citación del artículo: Chaparro-Herrera, S., Lopera-Salazar, A., Gutiérrez-Zuluaga, A. M., Betancur, J., Martínez Alvarado, D., Rivera Gutiérrez, H. F. \& Parra, J. L. (2019). Avifauna en dos complejos de páramo de Antioquia, Colombia. Biota Colombiana, 20(1), 91-105. DOI:10.21068/c2019.v20n01a06.

Recibido: 16 de mayo de 2018

Aceptado: 24 de enero de 2019 